\title{
Solving a well-posed fractional initial value problem by a complex approach
}

\author{
Arran Fernandez ${ }^{1 *}$ (D), Sümeyra Uçar ${ }^{2}$ and Necati Özdemir
}

\author{
"Correspondence: \\ arran.fernandez@emu.edu.tr \\ 'Department of Mathematics, \\ Faculty of Arts and Sciences, Eastern \\ Mediterranean University, \\ Famagusta, Northern Cyprus, via \\ Mersin 10, Turkey \\ Full list of author information is \\ available at the end of the article
}

\begin{abstract}
Nonlinear fractional differential equations have been intensely studied using fixed point theorems on various different function spaces. Here we combine fixed point theory with complex analysis, considering spaces of analytic functions and the behaviour of complex powers. It is necessary to study carefully the initial value properties of Riemann-Liouville fractional derivatives in order to set up an appropriate initial value problem, since some such problems considered in the literature are not well-posed due to their initial conditions. The problem that emerges turns out to be dimensionally consistent in an unexpected way, and therefore suitable for applications too.
\end{abstract}

MSC: 34A08; 34A12; 34M05

Keywords: Fractional differential equations; Initial value problems; Well-posedness; Fixed point theorems; Existence-uniqueness problems; Nonlinear differential equations

\section{Introduction}

Fractional differential equations are a generalisation of the usual differential equations in which the order of differentiation is allowed to be "fractional": that is to say, not only a natural number but any real or complex number. The operators of fractional differentiation and fractional integration have been widely studied, both for their pure mathematical properties [31,32,38] and for their applications in physics, biology, engineering, economics, etc. $[8,25,29,42]$. Many of the techniques used for classical differential equations, both ordinary and partial, can be extended to fractional differential equations. These include numerical methods $[5,33,43]$ and various types of analytical approaches $[2,4,6,11]$, including for fractional differential equations in more general settings such as manifolds or Banach spaces [30, 37, 41]. Several elementary fractional differential equations naturally give rise to special functions such as Mittag-Leffler functions and their generalisations as solutions $[3,26,28]$, and such functions have also been used as kernels in defining new fractional integral and derivative operators.

Complex analysis is a generalisation of real analysis in which variables and functions are allowed to take values in the complex plane. The ideas and methods of complex analysis have proven to be very important in certain analytical methods for differential equations:

(c) The Author(s) 2021. This article is licensed under a Creative Commons Attribution 4.0 International License, which permits use sharing, adaptation, distribution and reproduction in any medium or format, as long as you give appropriate credit to the original author(s) and the source, provide a link to the Creative Commons licence, and indicate if changes were made. The images or other third party material in this article are included in the article's Creative Commons licence, unless indicated otherwise in a credit line to the material. If material is not included in the article's Creative Commons licence and your intended use is not permitted by statutory regulation or exceeds the permitted use, you will need to obtain permission directly from the copyright holder. To view a copy of this licence, visit http://creativecommons.org/licenses/by/4.0/. 
for example, the Fokas method [19-21] relies heavily on Cauchy's theorem for deforming complex contour integrals, and the d-bar method $[1,22,23]$ is based on using the complex $\mathrm{d}$-bar derivative related to the Cauchy-Riemann equations. Some of these complex definitions and methods have recently been extended into fractional calculus, in some papers on complex methods for fractional differential equations $[9,12,17]$ and the very recent fractionalisation of the complex d-bar derivative [18], although historically the connections between complex analysis and fractional calculus have not been deeply explored. Therefore, all indications are that complex methods will be equally useful in fractional calculus as in classical calculus, but a lot of work still needs to be done in developing this area.

There are many different ways to define fractional derivatives and fractional integrals, leading to a rich and diverse field of study [10,27]. We shall focus primarily on the most classical and commonly used definition, which is called Riemann-Liouville. Here, fractional integrals are defined by

$$
I_{x}^{\alpha} f(x)=\frac{1}{\Gamma(\alpha)} \int_{0}^{x}(x-y)^{\alpha-1} f(y) \mathrm{d} y, \quad \operatorname{Re}(\alpha)>0,
$$

where $f$ is an $L^{1}$ function, or any function such that this expression is well defined, and $x$ is usually assumed to be contained in some interval $[0, X]$. Fractional derivatives can be seen as an extension of fractional integrals, the definition being

$$
D_{x}^{\alpha} f(x)=\frac{\mathrm{d}^{n}}{\mathrm{~d} x^{n}}\left(I^{n-\alpha} f(x)\right), \quad n:=\lfloor\operatorname{Re}(\alpha)\rfloor+1, \operatorname{Re}(\alpha) \geq 0,
$$

where $f$ is an $n$ times differentiable function. By adopting the notational convention $D_{x}^{\alpha} f(x)=I_{x}^{-\alpha} f(x)$, we now have a definition of $D_{x}^{\alpha} f(x)$ and of $I_{x}^{\alpha} f(x)$ for all $\alpha \in \mathbb{C}$, and these are analytic functions of $\alpha$ [38]. (Note that here we are using the convention that the "constant of integration" is taken to be 0 .)

In the context of complex analysis, it is sometimes more useful to use, instead of an integral directly from 0 to $x$, an integral that loops along a Hankel contour around $x$. In this context we replace $x$ with $z$ since it is a complex variable, and write the definition as follows [38, $\$ 22]$ :

$$
D_{z}^{\alpha} f(z)=\frac{\Gamma(1+\alpha)}{2 \pi i} \int_{\mathcal{L}_{z}}(w-z)^{-\alpha-1} f(w) \mathrm{d} w, \quad \alpha \in \mathbb{C} \backslash \mathbb{Z}^{-},
$$

where $\mathcal{L}_{z}$ is the Hankel-type contour which passes through 0 and wraps around the branch point $z$. This definition is equivalent to the standard Riemann-Liouville definition, but it works for both fractional integrals and fractional derivatives (one unified formula), and it is often more useful in cases where the variables involved are complex.

Having considered the basic setup and operators of fractional calculus, let us move on to the main problem under discussion for this work.

In some recent papers of Şan et al. [39, 40], some fractional initial value problems (IVPs) were studied from the complex point of view, and existence-uniqueness theorems were shown using the machinery of complex analysis. In general, the problems considered were of the following form:

$$
\begin{aligned}
& D_{z}^{\alpha} u(z)=f(z, u(z)), \\
& u(0)=b,
\end{aligned}
$$


where the function $f$ is given satisfying certain assumptions. Here, we shall consider carefully the nature of the initial conditions required for such problems, and in the end we shall solve a related problem with initial value conditions which guarantee a natural wellposedness.

To understand the problem with IVP (1)-(2), it is necessary to consider the extra condition connecting $f$ and $b$, namely

$$
\left[z^{\alpha} f(z, b)\right]_{z=0}=\frac{b}{\Gamma(1-\alpha)}
$$

(this is called condition II in [40] and condition (iv) in [39]), and also the equivalent formulation of the problem in Volterra form as

$$
u(z)=\frac{1}{\Gamma(\alpha)} \int_{0}^{z} \frac{f(\zeta, u(\zeta))}{(z-\zeta)^{1-\alpha}} \mathrm{d} \zeta
$$

(see Lemma 3.4 in [40] and Lemma 2.2 in [39]).

It should be noted that assumption (3) is a very strong one regarding the constant $b$. If we are given the function $f$, then there is likely to be only one possibility for $b$ which satisfies (3). This is already a warning sign: in classical IVPs, the differential equation and the initial condition are largely independent of each other, and the initial condition can be changed freely to obtain different solution functions.

Now consider the equivalent form (4). As it is shown in Lemma 3.4 of [40] and Lemma 2.2 of [39], this is precisely equivalent to IVP (1)-(2), given appropriate assumptions including (3). However, note that formulation (4) does not contain the initial value $b$ at all. In fact, assumption (3) ensures consistency of the Volterra form (4) with the initial condition (2):

$$
\begin{aligned}
\lim _{z \rightarrow 0}\left(\frac{1}{\Gamma(\alpha)} \int_{0}^{z} \frac{f(\zeta, u(\zeta))}{(z-\zeta)^{1-\alpha}} \mathrm{d} \zeta\right) & =\lim _{z \rightarrow 0}\left(\frac{1}{\Gamma(\alpha)} \int_{0}^{z} \frac{b}{\Gamma(1-\alpha)} \zeta^{-\alpha}(z-\zeta)^{\alpha-1} \mathrm{~d} \zeta\right) \\
& =\lim _{z \rightarrow 0}\left(\frac{b}{\Gamma(\alpha) \Gamma(1-\alpha)} \int_{0}^{1} w^{-\alpha}(1-w)^{\alpha-1} \mathrm{~d} w\right) \\
& =\frac{b}{\Gamma(\alpha) \Gamma(1-\alpha)} B(\alpha, 1-\alpha)=b,
\end{aligned}
$$

using the substitution $\zeta=z w$ and the definition of the beta function.

It is now clear that assumption (3) just means that the initial behaviour of $f$ is described by $b$, and so the two equations (1) and (2) are not truly independent of each other. The results of the papers $[39,40]$ are correct, but the IVPs being studied are not well-posed. Well-posedness requires that the solution depend continuously on the initial conditions, and here there is not even a continuous set of possible initial conditions for a given $f$. Condition (3) is too restrictive.

For this reason, we are here reconsidering the fractional differential equations studied by [39, 40]. After careful consideration, we associate the differential equation (1) with an initial condition different from (2). It is necessary to forget the assumption that $u$ should be analytic, since some nonsingular behaviour is required for a well-posed fractional IVP in this setting. Having set up the new form of IVP, we proceed to solve it using methods 
similar to those in $[39,40]$, proving existence and uniqueness of solutions using fixed point theorems.

We believe that our work will be an important contribution to the understanding of fractional initial value problems: not because of the methodology we have used, much of which is fairly routine, but because of our careful consideration of well-posedness leading to a new way of formulating fractional initial value problems. As well as the mathematical concern of well-posedness, there is also a physical concern of dimensionality, which must be considered carefully when fractional derivatives are involved. Both initial conditions $[34,36]$ and dimensionality $[7,24]$ are frequently discussed issues in fractional calculus, and our formulation presents a new way of solving both of these issues.

The structure of our paper is as follows. In Sect. 2 we derive and prove the main theorems of the paper: first figuring out an appropriate initial condition to associate with the differential equation (1), then proving existence and uniqueness in detail, and finally rewriting the whole initial value problem in such a way that the solution function is in a more natural function space and the problem is more likely to be useful in applications. In Sect. 3 we illustrate the results with several examples of different types, providing specific fractional initial value problems and conditions under which they have unique solutions. In Sect. 4 we conclude the paper with some remarks and ideas for future directions of research in this area.

\section{Main results}

\subsection{Deriving the Volterra form}

We will consider an initial value problem for the following nonlinear fractional differential equation:

$$
D_{z}^{\alpha} u(z)=f(z, u(z))
$$

where $\alpha \in(0,1)$ is fixed and the variable $z$ is in the open unit disc $D(0, R) \subset \mathbb{C}$.

What will be an appropriate initial condition to be combined with equation (5)?

We would like to rewrite the equation in the following Volterra-type form:

$$
u(z)=I_{z}^{\alpha}[f(z, u(z))]+(\text { initial-value term })
$$

by applying a fractional integral. This form of the problem will be more approachable using fixed point theory, since the fractional integral on the right-hand side can be suitably bounded in terms of certain conditions on $u$ and $f$.

So, what happens when we apply a fractional integral to both sides of equation (5)? In general, Riemann-Liouville fractional integrals and derivatives satisfy the following composition property [38, Eq. (2.61)] for $\alpha \in(0,1)$ :

$$
I_{z}^{\alpha} D_{z}^{\alpha} u(z)=u(z)-\frac{z^{\alpha-1}}{\Gamma(\alpha)}\left[I_{z}^{1-\alpha} u(z)\right]_{z=0} .
$$

The initial value term on the right-hand side is a fractional integral evaluated at its starting point:

$$
\left[I_{z}^{1-\alpha} u(z)\right]_{z=0}=\lim _{z \rightarrow 0}\left(\frac{1}{\Gamma(1-\alpha)} \int_{0}^{z}(z-w)^{-\alpha} u(w) \mathrm{d} w\right)
$$


If the function $u$ is suitably well behaved (e.g. bounded in the neighbourhood of 0 ), then this integral will necessarily be zero. But this is not the desired behaviour: if the initial value term is always zero, then there is no well-posed problem to be created from equation (5) with an initial condition.

Therefore, we shall assume a different type of condition on $u$. In fact, we want $u(z)$ to be singular at $z=0$. We observe the following fact:

$$
I_{z}^{1-\alpha}\left(z^{\alpha-1}\right)=\frac{\Gamma(\alpha)}{\Gamma(1)} z^{0}=\Gamma(\alpha) .
$$

Since this function is constant, we also have

$$
\lim _{z \rightarrow 0}\left(I_{z}^{1-\alpha}\left(z^{\alpha-1}\right)\right)=\Gamma(\alpha)
$$

This demonstrates that a fractional integral taken from 0 to $z$, evaluated at $z=0$, is not necessarily zero. Furthermore, for any function $u(z)$ such that $u(z) \sim k z^{\alpha-1}$ as $z \rightarrow 0(k$ constant), we have

$$
\lim _{z \rightarrow 0}\left(I_{z}^{1-\alpha} u(z)\right)=k \Gamma(\alpha)
$$

Note that any such function $u$ will not be analytic in any punctured neighbourhood of 0 . However, its lack of analyticity is well understood, since we know the fractional "valency" of the function at 0 .

From the above work, we now understand how to turn equation (5) into a well-posed initial value problem which is equivalent to a Volterra type equation of the form (6). The result is given as follows.

Theorem 2.1 Let $\alpha \in(0,1)$ and $k \in \mathbb{C}$ be given. Let $f$ be a function of two variables satisfying the assumption

(A1) $f\left(z, k z^{\alpha-1}\right)=o\left(z^{-1}\right)$ as $z \rightarrow 0$.

Then the following initial value problem

$$
\begin{aligned}
& D_{z}^{\alpha} u(z)=f(z, u(z)), \\
& \lim _{z \rightarrow 0}\left[z^{1-\alpha} u(z)\right]=k,
\end{aligned}
$$

is equivalent to the following Volterra type integral equation:

$$
u(z)=I_{z}^{\alpha}[f(z, u(z))]+k z^{\alpha-1}
$$

in other words, a function $u$ is a solution of (9)-(10) if and only if it is a solution of (11).

Proof Firstly, assume that the initial value problem (9)-(10) is satisfied. Apply the fractional integral operator $I_{z}^{\alpha}$ to both sides of (9), and use identity (7) to get

$$
u(z)-\frac{z^{\alpha-1}}{\Gamma(\alpha)}\left[I_{z}^{1-\alpha} u(z)\right]_{z=0}=I_{z}^{\alpha}[f(z, u(z))]
$$


By the initial condition (10), we have $u(z) \sim k z^{\alpha-1}$ as $z \rightarrow 0$, and so equation (8) applies. Thus we have

$$
u(z)-k z^{\alpha-1}=I_{z}^{\alpha}[f(z, u(z))]
$$

which is equation (11).

For the converse, we start from the Volterra form (11). Applying the fractional derivative operator $D_{z}^{\alpha}$ to both sides, and noting that $D_{z}^{\alpha}\left(z^{\alpha-1}\right)=0$, we find

$$
D_{z}^{\alpha} u(z)=D_{z}^{\alpha} I_{z}^{\alpha}[f(z, u(z))]+0 .
$$

Since the fractional derivative of a fractional integral satisfies a semigroup law, this gives equation (9). Finally, since $I_{z}^{\alpha} z^{\beta}$ is only well defined for $\beta>-1$, we have, as $z \rightarrow 0$, $f(z, u(z))=o\left(z^{-1}\right)$, and so $I_{z}^{\alpha}[f(z, u(z))]=o\left(z^{\alpha-1}\right)$. Multiplying the given equation (11) by $z^{1-\alpha}$ and letting $z \rightarrow 0$, we get

$$
z^{1-\alpha} u(z)=z^{1-\alpha} I_{z}^{\alpha}[f(z, u(z))]+k \rightarrow 0+k \quad \text { as } z \rightarrow 0,
$$

which is exactly (10).

From the above theorem, we have a well-posed initial value problem and an equivalent Volterra form for it. The reason we sought this Volterra form was in order to apply fixed point theory to the integral. Let us then consider equation (11) in a more explicit form:

$$
u(z)=\frac{1}{\Gamma(\alpha)} \int_{0}^{z}(z-w)^{\alpha-1} f(w, u(w)) \mathrm{d} w+k z^{\alpha-1}
$$

The obvious way to proceed now would be to define a functional operator $P$ by

$$
(P u)(z):=\frac{1}{\Gamma(\alpha)} \int_{0}^{z}(z-w)^{\alpha-1} f(w, u(w)) \mathrm{d} w+k z^{\alpha-1},
$$

and try to show that this $P$ has a unique fixed point in an appropriate function space. However, the right-hand side of (12) contains a term $k z^{\alpha-1}$ which is unbounded in any neighbourhood of $z=0$, as is the function $u(z)$ itself. We would like to define our operator on a 'nice' Banach space. It makes more sense, then, to consider the function

$$
v(z)=z^{1-\alpha} u(z)-k,
$$

which is analytic near $z=0$ and equal to zero at $z=0$, and define instead a functional operator $Q$ by

$$
(Q v)(z):=\frac{z^{1-\alpha}}{\Gamma(\alpha)} \int_{0}^{z}(z-w)^{\alpha-1} f\left(w, w^{\alpha-1}[v(w)+k]\right) \mathrm{d} w
$$

The problem now reduces to finding suitable conditions on $f$ and a suitable function space for $v$, such that this operator $Q$ has a unique fixed point. 
Remark 2.2 If the analytic function $v(z)$ represents some real-life quantity $V$, then the dimensions of $u(z)=z^{\alpha-1}[v(z)+k]$ will be $(\operatorname{dim} z)^{\alpha-1} \cdot \operatorname{dim} V$, and the dimensions of the quantity $D_{z}^{\alpha} u(z)$ appearing in the fractional differential equation will be $\frac{\operatorname{dim} V}{\operatorname{dim} z}$. This is interesting because we can then get a fractional differential equation without involving fractional dimensions.

Some authors $[16,35]$ have mentioned the appearance of fractional dimensions as an argument against fractional calculus in applications. Sometimes this issue is resolved by using dimensioned constants to ensure dimensional consistency, but here we see a different type of resolution: a differential equation which uses fractional derivatives but has dimensionality like a 1st-order differential equation.

It is interesting to see how the mathematical and real-world considerations align well here. Above we used a mathematical argument to see that, for this problem, it is better not to take the fractional derivative of an analytic function but instead to apply $D_{z}^{\alpha}$ to a function which is $z^{\alpha-1}$ times an analytic function. Now we see that the same conclusion could emerge from considering the physical dimensionality of the analytic function involved.

Remark 2.3 The idea of considering fractional differential equations with fractional power-function behaviour in the nonlinear term is not a new one. Even some recent papers such as [13-15] have considered problems of this form; however, they used standard $C$ and $L^{p}$ function spaces. Our novelty is to work in the complex-analysis viewpoint, which is a much stronger restriction on the functions involved.

\subsection{Proving existence and uniqueness}

Definition 2.4 Let $R$ be a fixed positive real number. For the purposes of this section, we shall consider the space of functions $v(z)$ which are analytic on the disc $D(0, R)$ in the complex plane and satisfy $v(0)=1$. Let us denote this space by $\mathcal{A}$, and note that it a Banach space under the uniform norm $\|v\|=\sup _{z \in D(0, R)}|v(z)|$.

Theorem 2.5 Assume the following conditions on the function $f$ :

(A2) The expression $f\left(z, k z^{\alpha-1}\right)$ is an analytic function of $z$.

(A3) There exist positive constants $c$ and $\varepsilon$ such that $\frac{c R^{\varepsilon} \Gamma(\varepsilon)}{\Gamma(\alpha+\varepsilon)}<1$, and for all $z, \eta, v$,

$$
|f(z, \eta)-f(z, v)|<\frac{c}{|z|^{\alpha}}|\eta-v||z|^{\varepsilon} .
$$

Then there is a unique function $v(z)$ in the space $\mathcal{A}$ defined in Definition 2.4 such that $(Q v)(z)=v(z)$.

Proof From inequality (14), we have in particular

$$
\left|f\left(w, w^{\alpha-1}[v(w)+k]\right)\right|<c|w|^{\varepsilon-1}|v(w)|+\left|f\left(w, k w^{\alpha-1}\right)\right| .
$$


We will use this in the following work, starting from definition (13) of the operator Q:

$$
\begin{aligned}
& |Q v(z)|=\frac{|z|^{1-\alpha}}{\Gamma(\alpha)}\left|\int_{0}^{z}(z-w)^{\alpha-1} f\left(w, w^{\alpha-1}[v(w)+k]\right) \mathrm{d} w\right| \\
& \leq \frac{|z|^{1-\alpha}}{\Gamma(\alpha)} \int_{0}^{|z|}|z-w|^{\alpha-1}\left|f\left(w, w^{\alpha-1}[v(w)+k]\right)\right| \mathrm{d}|w| \\
& \leq \frac{|z|^{1-\alpha}}{\Gamma(\alpha)} \int_{0}^{|z|}|z-w|^{\alpha-1}\left(c|w|^{\varepsilon-1}|v(w)|+\left|f\left(w, k w^{\alpha-1}\right)\right|\right) \mathrm{d}|w| \\
& =\frac{|z|^{1-\alpha}}{\Gamma(\alpha)} c \int_{0}^{|z|}|z-w|^{\alpha-1}|w|^{\varepsilon-1}|v(w)| \mathrm{d}|w| \\
& +\frac{|z|^{1-\alpha}}{\Gamma(\alpha)} \int_{0}^{|z|}|z-w|^{\alpha-1}\left|f\left(w, k w^{\alpha-1}\right)\right| \mathrm{d}|w| \\
& \leq \frac{|z|^{1-\alpha}}{\Gamma(\alpha)} c\|v\| \int_{0}^{|z|}|z-w|^{\alpha-1}|w|^{\varepsilon-1} \mathrm{~d}|w| \\
& +\frac{|z|^{1-\alpha}}{\Gamma(\alpha)} \sup _{w}\left|f\left(w, k w^{\alpha-1}\right) w^{1-\varepsilon}\right| \int_{0}^{|z|}|z-w|^{\alpha-1}|w|^{\varepsilon-1} \mathrm{~d}|w| \\
& =\frac{|z|^{1-\alpha}}{\Gamma(\alpha)}\left(c\|v\|+\sup _{w}\left|f\left(w, k w^{\alpha-1}\right) w^{1-\varepsilon}\right|\right) \int_{0}^{1}|z-t z|^{\alpha-1}|t z|^{\varepsilon-1}|z| \mathrm{d} t \\
& =\frac{|z|^{1-\alpha}}{\Gamma(\alpha)}\left(c\|v\|+\sup _{w}\left|f\left(w, k w^{\alpha-1}\right) w^{1-\varepsilon}\right|\right)|z|^{\alpha-\varepsilon-1} B(\alpha, \varepsilon) \\
& =\frac{\Gamma(\varepsilon)}{\Gamma(\alpha+\varepsilon)}|z|^{\varepsilon}\left(c\|v\|+\sup _{w}\left|f\left(w, k w^{\alpha-1}\right) w^{1-\varepsilon}\right|\right) .
\end{aligned}
$$

This shows that the function $Q v$ is still bounded on the disc $D(0, R)$. Rewriting definition (13) of $Q$ as

$$
(Q v)(z)=\frac{1}{\Gamma(\alpha)} \int_{0}^{z}\left(1-\frac{w}{z}\right)^{\alpha-1} f\left(w, w^{\alpha-1}[v(w)+k]\right) \mathrm{d} w
$$

we see that $Q v(z)$ is an analytic function of $z$ at all points $z \neq 0$. (This is because $1-\frac{w}{z} \in$ $D(1,1)$, and therefore its fractional power can still be an analytic function, while the $f$ part of the integrand is uniformly bounded by assumption (A3).) To ensure that we have an operator $Q: \mathcal{A} \rightarrow \mathcal{A}$, we just need to check the behaviour near $z=0$. As $z \rightarrow 0$, we know that $v(z) \rightarrow 0$, and so

$$
(Q v)(z) \sim \frac{z^{1-\alpha}}{\Gamma(\alpha)} \int_{0}^{z}(z-w)^{\alpha-1} f\left(w, k w^{\alpha-1}\right) \mathrm{d} w .
$$

Following the original assumption (A1) on the function $f\left(w, k w^{\alpha-1}\right)$, let us try writing $f\left(w, k w^{\alpha-1}\right) \sim C w^{\beta}$ as $w \rightarrow 0$ with $\operatorname{Re}(\beta)>-1$. Then

$$
\begin{aligned}
(Q v)(z) & \sim \frac{C}{\Gamma(\alpha)} z^{1-\alpha} \int_{0}^{z}(z-w)^{\alpha-1} w^{\beta} \mathrm{d} w \\
& =\frac{C}{\Gamma(\alpha)} z^{1-\alpha} \int_{0}^{1}(z-z t)^{\alpha-1}(z t)^{\beta} z \mathrm{~d} t \\
& =\frac{C}{\Gamma(\alpha)} z^{1-\alpha} z^{\alpha+\beta-1} B(\alpha, \beta)=\frac{C \Gamma(\beta)}{\Gamma(\alpha+\beta)} z^{\beta} .
\end{aligned}
$$


We need $Q v(z)$ to be an analytic function of $z$, therefore we should have $\beta \in \mathbb{Z}_{0}^{+}$. Then the assumption $f\left(w, k w^{\alpha-1}\right) \sim C w^{\beta}$ becomes precisely that $f\left(w, k w^{\alpha-1}\right)$ should be analytic near $w=0$. This is the motivation for assumption (A2).

Now we know that $Q$ is a well-defined operator on the Banach space $\mathcal{A}$. It remains to show that it is a contraction.

$$
\begin{aligned}
& \left|Q v(z)-Q v_{0}(z)\right| \\
& \quad=\left|\frac{z^{1-\alpha}}{\Gamma(\alpha)} \int_{0}^{z}(z-w)^{\alpha-1}\left(f\left(w, w^{\alpha-1}[v(w)+k]\right)-f\left(w, w^{\alpha-1}\left[v_{0}(w)+k\right]\right)\right) \mathrm{d} w\right| \\
& \quad \leq \frac{|z|^{1-\alpha}}{\Gamma(\alpha)} \int_{0}^{|z|}|z-w|^{\alpha-1}\left|f\left(w, w^{\alpha-1}[v(w)+k]\right)-f\left(w, w^{\alpha-1}\left[v_{0}(w)+k\right]\right)\right| \mathrm{d}|w| \\
& \quad \leq \frac{|z|^{1-\alpha}}{\Gamma(\alpha)} \int_{0}^{|z|}|z-w|^{\alpha-1} \frac{c}{|w|^{\alpha-\varepsilon}}\left|w^{\alpha-1}[v(w)+k]-w^{\alpha-1}\left[v_{0}(w)+k\right]\right| \mathrm{d}|w| \\
& \quad=\frac{|z|^{1-\alpha}}{\Gamma(\alpha)} \int_{0}^{|z|}|z-w|^{\alpha-1} c|w|^{\varepsilon-1}\left|v(w)-v_{0}(w)\right| \mathrm{d}|w| \\
& \quad \leq \frac{|z|^{1-\alpha}}{\Gamma(\alpha)} c\left\|v-v_{0}\right\| \int_{0}^{|z|}|z-w|^{\alpha-1}|w|^{\varepsilon-1} \mathrm{~d}|w| \\
& \quad=\frac{|z|^{1-\alpha}}{\Gamma(\alpha)} c\left\|v-v_{0}\right\||z|^{\alpha+\varepsilon-1} B(\alpha, \varepsilon)=\frac{c \Gamma(\varepsilon)}{\Gamma(\alpha+\varepsilon)}\left\|v-v_{0}\right\||z|^{\varepsilon} .
\end{aligned}
$$

By the assumption that $\frac{c R^{\varepsilon} \Gamma(\varepsilon)}{\Gamma(\alpha+\varepsilon)}<1$, this means $Q$ is a contraction on the space $\mathcal{A}$. By the contraction mapping theorem, therefore, $Q$ has a unique fixed point in $\mathcal{A}$.

Remark 2.6 It is important to state explicitly that assumption (A2) on $f$ directly implies the previous assumption (A1) used in Theorem 2.1. Therefore (A2) is a stronger condition.

Theorem 2.7 Let $\alpha \in(0,1), k \in \mathbb{C}$, and $R \in \mathbb{R}^{+}$be given. Let $f$ be a function of two variables satisfying assumptions $(A 2)$ and $(A 3)$ stated in Theorem 2.5 . Then the initial value problem (9)-(10) has a unique solution $u(z)$ satisfying the condition that the function $z^{1-\alpha} u(z)$ should be analytic on the disc $D(0, R)$ in the complex plane.

Proof Since assumption (A2) implies assumption (A1), the result of Theorem 2.1 holds, i.e. solving the initial value problem (9)-(10) is equivalent to solving the Volterra-type equation (11). And we saw that this equation

$$
u(z)=I_{z}^{\alpha}[f(z, u(z))]+k z^{\alpha-1}
$$

is equivalent to the following equation in $v(z)=z^{1-\alpha} u(z)-k$ :

$$
v(z)=I_{z}^{\alpha}\left[f\left(z, z^{\alpha-1}[v(z)+k]\right)\right] .
$$

By Theorem 2.5, this equation has a unique solution $v(z)$ which is analytic on $D(0, R)$ and equal to zero at $z=0$. Therefore there is a unique solution $u(z)$ such that $z^{1-\alpha} u(z)$ is analytic on $D(0, R)$ (being equal to $k$ at $z=0$ follows from condition (10) that is part of the initial value problem). 
Following the ideas discussed in Remark 2.2, we can also rewrite the initial value problem in such a way that the differential equation is less elementary but the initial condition is much simpler and more natural.

Theorem 2.8 Let $\alpha \in(0,1), k \in \mathbb{C}$, and $R \in \mathbb{R}^{+}$be given. Let $g$ be a function of two variables satisfying the assumptions

(A4) $g$ is analytic as a function of its first parameter.

(A5) There exist positive constants $c$ and $\varepsilon$ such that $\frac{c R^{\varepsilon} \Gamma(\varepsilon)}{\Gamma(\alpha+\varepsilon)}<1$, and for all $z, \eta, v$,

$$
|g(z, \eta)-g(z, v)|<c|z|^{\varepsilon-1}|\eta-v|
$$

Then the initial value problem

$$
\begin{aligned}
& D_{z}^{\alpha}\left[z^{\alpha-1} q(z)\right]=g(z, q(z)), \\
& q(0)=k,
\end{aligned}
$$

has a unique solution $q(z)$ that is analytic on the $\operatorname{disc} D(0, R) \subset \mathbb{C}$.

Proof This follows from the previous theorem when we write $q(z)=z^{1-\alpha} u(z)$ and $g(z, w)=$ $f\left(z, z^{\alpha-1} w\right)$. Assumptions (A4) and (A5) are exactly the same as the previous ones (A2) and (A3) under this substitution.

Remark 2.9 Following on from Remark 2.2, we note that the initial value problem (16)(17) is in a form which is dimensionally elegant: the left-hand side has dimensions $\frac{\operatorname{dim} q}{\operatorname{dim} z}$, and for appropriate functions $g$, the right-hand side would have the same dimensions. Furthermore, the initial condition (17) is in a natural form, the same indeed as the initial condition (2) used by Şan; however, the additional power function compared with Şan's differential equation (1) ensures the well-posedness of our problem (16)-(17) considered here.

\section{Examples}

In this section, we present some examples to illustrate the results obtained in general above. In each case, a particular function $g(z, w)$ is given for use in Theorem 2.8, conditions (A4) and (A5) are verified to be true, and then the result is stated in the case of this particular choice of function $g$.

Example 3.1 Let $g(z, w)=z^{2}+w z$. It is clear that $g$ is an analytic function of its first parameter, so (A4) is satisfied. For condition (A5), let $\varepsilon=2$ and choose any $c>1$, since for all $z, \eta, v$ we have

$$
|g(z, \eta)-g(z, v)|=\left|z^{2}+\eta z-z^{2}-v z\right|=|z||\eta-v|<c|z|^{2-1}|\eta-v| .
$$

The chosen $\varepsilon$ and $c$ should satisfy $\frac{c R^{\varepsilon} \Gamma(\varepsilon)}{\Gamma(\alpha+\varepsilon)}=\frac{c R^{2}}{\Gamma(\alpha+2)}<1$, i.e. $R<\sqrt{\frac{\Gamma(2+\alpha)}{c}}$.

Therefore, the initial value problem

$$
\begin{aligned}
& D_{z}^{\alpha}\left[z^{\alpha-1} q(z)\right]=z^{2}+z q(z), \\
& q(0)=k
\end{aligned}
$$

has a unique analytic solution $q$ on the $\operatorname{disc} D(0, \sqrt{\Gamma(2+\alpha)}) \subset \mathbb{C}$. 
Example 3.2 Let $g(z, w)=\sqrt{e^{z}+1}+w z$. This is an analytic function of its first parameter on the disc $D(0, \pi)$, assuming we choose the principal (horizontal) branch cuts from the branch points at $z=i \pi$ and $z=-i \pi$. Thus condition (A4) is satisfied with $R<\pi$. For condition (A5), let $\varepsilon=2$ and choose any $c>1$, since for all $z, \eta, v$ we have

$$
\begin{aligned}
|g(z, \eta)-g(z, v)| & =\left|\sqrt{e^{z}+1}+\eta z-\sqrt{e^{z}+1}-v z\right| \\
& =|z||\eta-v|<c|z|^{2-1}|\eta-v| .
\end{aligned}
$$

As before, the condition $\frac{c R^{\varepsilon} \Gamma(\varepsilon)}{\Gamma(\alpha+\varepsilon)}<1$ gives $R<\sqrt{\frac{\Gamma(2+\alpha)}{c}}<\Gamma(2+\alpha)$. Since it is assumed $\alpha \in$ $(0,1)$, the maximum possible value of $\Gamma(2+\alpha)$ is $\Gamma(3)=2$, so the assumption $R<\pi$ is also automatically satisfied.

Therefore, the initial value problem

$$
\begin{aligned}
& D_{z}^{\alpha}\left[z^{\alpha-1} q(z)\right]=\sqrt{e^{z}+1}+z q(z), \\
& q(0)=k
\end{aligned}
$$

has a unique analytic solution $q$ on the $\operatorname{disc} D(0, \sqrt{\Gamma(2+\alpha)}) \subset \mathbb{C}$.

Example 3.3 Let $g(z, w)=z+w \sqrt{z+a}$. This is an analytic function of its first parameter on the disc $D(0, a)$, assuming we choose the principal branch for the function $\sqrt{z+a}$. Thus condition (A4) is satisfied with $R<a$. For condition (A5), let $\varepsilon=\frac{3}{2}$ and $c=\sqrt{2}$, since for all $\eta, v$ and all $z \in D(0, a)$ we have

$$
\begin{aligned}
|g(z, \eta)-g(z, v)| & =|z-\eta \sqrt{z+a}-z-v \sqrt{z+a}| \\
& =|z+a|^{1 / 2}|\eta-v|<(2|z|)^{1 / 2}|\eta-v|=\sqrt{2}|z|^{\frac{3}{2}-1}|\eta-v| .
\end{aligned}
$$

The chosen $\varepsilon$ and $c$ should satisfy

$$
1>\frac{c R^{\varepsilon} \Gamma(\varepsilon)}{\Gamma(\alpha+\varepsilon)}=\frac{\sqrt{2 R^{3}} \Gamma(3 / 2)}{\Gamma(\alpha+3 / 2)}=\frac{\sqrt{\pi R^{3}}}{\sqrt{2} \Gamma(\alpha+3 / 2)},
$$

i.e. $\pi R^{3}<2 \Gamma\left(\alpha+\frac{3}{2}\right)^{2}$ or $R<\left(\frac{2}{\pi} \Gamma\left(\alpha+\frac{3}{2}\right)^{2}\right)^{1 / 3}$. Since it is assumed $\alpha \in(0,1)$, the maximum possible value of $\Gamma\left(\alpha+\frac{3}{2}\right)$ is $\Gamma(5 / 2)=3 \sqrt{\pi} / 4$, giving $R<(9 / 8)^{1 / 3}=\sqrt[3]{9} / 2$. So the assumption $R<a$ is also automatically satisfied for any $a \geq \sqrt[3]{9} / 2$.

Therefore, the initial value problem

$$
\begin{aligned}
& D_{z}^{\alpha}\left[z^{\alpha-1} q(z)\right]=z+q(z) \sqrt{z+a}, \\
& q(0)=k
\end{aligned}
$$

has a unique analytic solution $q$ on the $\operatorname{disc} D\left(0, \sqrt[3]{\frac{2}{\pi} \Gamma\left(\alpha+\frac{3}{2}\right)^{2}}\right) \subset \mathbb{C}$, provided the constant $a$ is at least $\sqrt[3]{9} / 2$.

\section{Conclusions and further work}

In this paper, we have refined the recent work of Şan $[39,40]$ on complex fixed point theorems for fractional initial value problems, replacing the initial conditions used there by 
new initial conditions which are more appropriate for the fractional problems. Having established a fractional initial value problem which is well-posed for solutions, we proceeded to prove existence and uniqueness results in a space of complex analytic functions by using Banach's fixed point theorem (the contraction mapping theorem).

Our particular choice of initial value problem is interesting because it is not an obvious choice from either the mathematical or physical point of view, but deeper analysis shows that it is indeed a natural choice: both for making a mathematically well-posed problem, and physically for dimensional consistency. In particular, Theorem 2.8 gives a new formulation of a fractional differential equation which is unexpected but dimensionally consistent and with a mathematically well-behaved solution. It is our hope that this idea may have some influence in the future study of fractional initial value problems.

We illustrated our general results by some examples using particular functions, in which some curious bounds and constants arose from the required conditions.

In the future, we intend to extend the results of this paper by considering other types of fractional calculus. The work here is set within the Riemann-Liouville model, but it may be possible to extend it, applying the same arguments in some general class of fractional operators, in order to obtain further results which would be useful in different types of modelling problems.

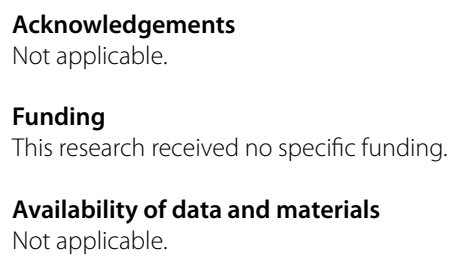

\section{Publisher's Note}

Springer Nature remains neutral with regard to jurisdictional claims in published maps and institutional affiliations.

Received: 3 December 2020 Accepted: 2 May 2021 Published online: 28 May 2021

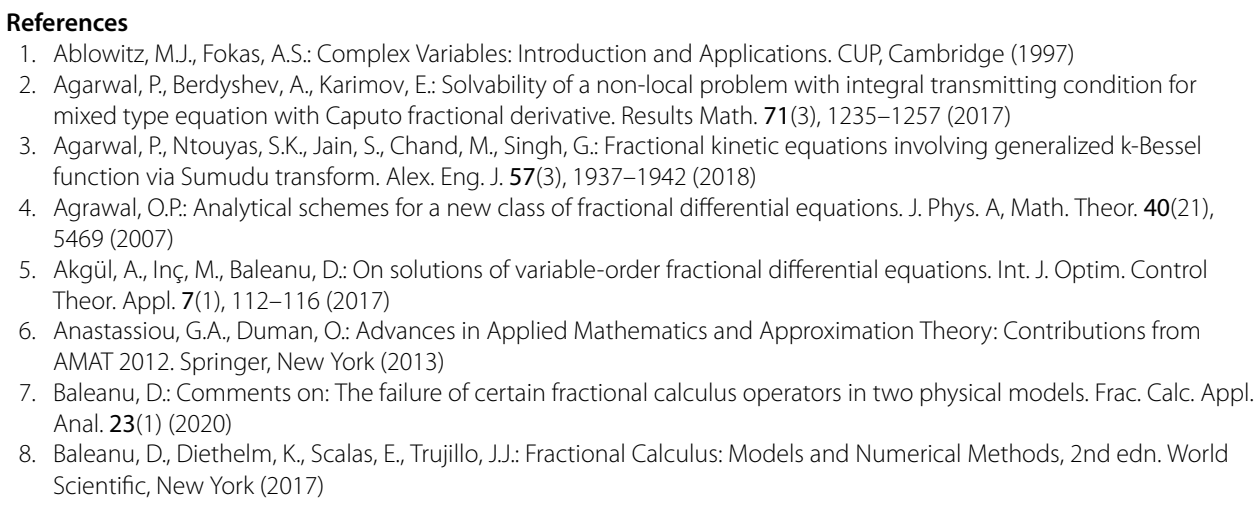

5. Akgül, A., Inç, M., Baleanu, D.: On solutions of variable-order fractional differential equations. Int. J. Optim. Control Theor. Appl. 7(1), 112-116 (2017)

6. Anastassiou, G.A., Duman, O.: Advances in Applied Mathematics and Approximation Theory: Contributions from AMAT 2012. Springer, New York (2013)

7. Baleanu, D.: Comments on: The failure of certain fractional calculus operators in two physical models. Frac. Calc. Appl. Anal. 23(1) (2020)

8. Baleanu, D., Diethelm, K., Scalas, E., Trujillo, J.J.: Fractional Calculus: Models and Numerical Methods, 2nd edn. World Scientific, New York (2017) 
9. Baleanu, D., Fernandez, A.: A generalisation of the Malgrange-Ehrenpreis theorem to find fundamental solutions to fractional PDEs. Electron. J. Qual. Theory Differ. Equ. 2017, 15 (2017)

10. Baleanu, D., Fernandez, A.: On fractional operators and their classifications. Mathematics 7(9), 830 (2019)

11. Baltaeva, U., Agarwal, P.: Boundary-value problems for the third-order loaded equation with noncharacteristic type-change boundaries. Math. Methods Appl. Sci. 41(9), 3307-3315 (2018)

12. Başkonuş, H.M., Bulut, H.: New complex exact travelling wave solutions for the generalized Zakharov equation with complex structures. Int. J. Optim. Control Theor. Appl. 6(2), 141-150 (2016)

13. Cabada, A., Kisela, T.: Existence of positive periodic solutions of some nonlinear fractional differential equations. Commun. Nonlinear Sci. Numer. Simul. 50, 51-67 (2017)

14. Cabada, A., Wanassi, O.K.: Existence results for nonlinear fractional problems with non-homogeneous integral boundary conditions. Mathematics 8(2), 255 (2020)

15. Cabada, A., Wanassi, O.K.: Existence and uniqueness of positive solutions for nonlinear fractional mixed problems. arXiv:1903.09042. Preprint

16. Dokoumetzidis, A., Magin, R., Macheras, P.: A commentary on fractionalization of multi-compartmental models. J. Pharmacokinet. Pharmacodyn. 37, 203-207 (2010)

17. Fernandez, A., Baleanu, D., Fokas, A.S.: Solving PDEs of fractional order using the unified transform method. Appl. Math. Comput. 339C, 738-749 (2018)

18. Fernandez, A., Bouzouina, C.: Fractionalisation of complex d-bar derivatives. Complex Var. Elliptic Equ. 66, 437-475 (2021)

19. Fokas, A.S.: A unified transform method for solving linear and certain nonlinear PDEs. Proc. R. Soc. Lond. A 453, 1411-1443 (1997)

20. Fokas, A.S.: Two-dimensional linear partial differential equations in a convex polygon. Proc. R. Soc. Lond. A 457, 371-393 (2001)

21. Fokas, A.S.: A Unified Approach to Boundary Value Problems. SIAM, Philadelphia (2008)

22. Fokas, A.S., Pinotsis, D.A.: The Dbar formalism for certain linear non-homogeneous elliptic PDEs in two dimensions. Eur. J. Appl. Math. 17, 323-346 (2006)

23. Fokas, A.S., van der Weele, M.C.: Complexification and integrability in multidimensions. J. Math. Phys. 59, 091413 (2018)

24. Gómez-Aguilar, J.F., Baleanu, D.: Fractional transmission line with losses. Z. Naturforsch. A 69(10-11), 539-546 (2015)

25. Hilfer, R. (ed.): Applications of Fractional Calculus in Physics World Scientific, Singapore (2000)

26. Huseynov, I.T., Ahmadova, A., Fernandez, A., Mahmudov, N.I.: Explicit analytic solutions of incommensurate fractional differential equation systems. Appl. Math. Comput. 390C, 125590 (2021)

27. Kochubei, A., Luchko, Y. (eds.): Handbook of Fractional Calculus with Applications, Volume 1: Basic Theory de Gruyter, Berlin (2019)

28. Luchko, Y.F., Gorenflo, R.: An operational method for solving fractional differential equations. Acta Math. Vietnam. 24, 207-234 (1999)

29. Magin, R.L.: Fractional Calculus in Bioengineering. Begell House Publishers, Connecticut (2006)

30. Mahmudov, N.I.: Approximate controllability of fractional neutral evolution equations in Banach spaces. Abstr. Appl. Anal. 2013, 531894 (2013)

31. Miller, K.S., Ross, B.: An Introduction to the Fractional Calculus and Fractional Differential Equations. Wiley, New York (1993)

32. Oldham, K.B., Spanier, J.: The Fractional Calculus. Academic Press, San Diego (1974)

33. Özdemir, N., Uçar, S., Eroğlu, B.B.I.: Dynamical analysis of fractional order model for computer virus propagation with kill signals. Int. J. Nonlinear Sci. Numer. Simul. 21(3-4), 239-247 (2020)

34. Sabatier, J.: Fractional-order derivatives defined by continuous kernels: are they really too restrictive? Fractal Fract. 4(3), 40 (2020)

35. Sabatier, J., Farges, C., Tartaglione, V.: Some alternative solutions to fractional models for modelling power law type long memory behaviours. Mathematics 8(2), 196 (2020)

36. Sabatier, J., Merveillaut, M., Malti, R., Oustaloup, A.: How to impose physically coherent initial conditions to a fractional system? Commun. Nonlinear Sci. Numer. Simul. 15(5), 1318-1326 (2010)

37. Salem, H.A.H.: Hadamard-type fractional calculus in Banach spaces. Rev. R. Acad. Cienc. Exactas Fís. Nat., Ser. A Mat. $113(2), 987-1006(2019)$

38. Samko, S.G., Kilbas, A.A., Marichev, O.I.: Fractional Integrals and Derivatives: Theory and Applications. Gordon \& Breach Science Publishers, Yverdon (1993) Originally in Russian, Nauka i Tekhnika, Minsk, 1987

39. San, M.: Complex variable approach to the analysis of a fractional differential equation in the real line. C. R. Math. 356 293-300 (2018)

40. Şan, M., Soltanov, K.N.: The new existence and uniqueness results for complex nonlinear fractional differential equation. arXiv:1512.04780. Preprint

41. Saoudi, K., Agarwal, P., Kumam, P., Ghanmi, A., Thounthong, P.: The Nehari manifold for a boundary value problem involving Riemann-Liouville fractional derivative. Adv. Differ. Equ. 2018(1), 1 (2018)

42. Sun, H.G., Zhang, Y., Baleanu, D., Chen, W., Chen, Y.Q.: A new collection of real world applications of fractional calculus in science and engineering. Commun. Nonlinear Sci. Numer. Simul. 64, 213-231 (2018)

43. Yavuz, M.: Novel solution methods for initial boundary value problems of fractional order with conformable differentiation. Int. J. Optim. Control Theor. Appl. 8(1), 1-7 (2018) 